\title{
Electrical signals induced in detectors by cosmic rays: a reciprocal look at electrodynamics
}

\author{
Philipp Windischhofer ${ }^{a * *}$ and Werner Riegler $^{b}$ \\ ${ }^{a}$ University of Oxford, Department of Physics, Oxford, United Kingdom \\ ${ }^{b}$ CERN, Geneva, Switzerland \\ E-mail: philipp.windischhofer@physics.ox.ac.uk, werner.riegler@cern.ch
}

\begin{abstract}
The computation of the radio emissions produced by an extended air shower at the location of the detector is a fundamental problem in the indirect detection of cosmic rays. Conventionally, simulation programs compute and sum the contributions from all particles in the shower, and then propagate this field through the environment and the signal processing chain of the experiment. This contribution presents a complementary formulation, which encodes all properties of the detector and its environment in a specially constructed electric field distribution, the "weighting field". Once the weighting field is known for a particular situation, the electrical signal produced in the detector can be computed very efficiently by convolving the weighting field with the trajectories of the charged particles in the shower.
\end{abstract}

$37^{\text {th }}$ International Cosmic Ray Conference (ICRC 2021)

July 12th - 23rd, 2021

Online - Berlin, Germany

\footnotetext{
*Presenter
} 


\section{Introduction}

A precise understanding of the electromagnetic emissions produced by cosmic ray-induced showers is critical for the design of sensitive cosmic ray radio experiments, as well as for the analysis of the data these detectors produce. The fidelity of Monte Carlo-based simulations of such showers and their radio signatures has improved enormously in recent years. These simulations typically proceed in two stages: once the trajectories of the particles generating the shower have been determined according to the shower model, commonly used tools, such as REAS [1] or ZHaireS [2] proceed with the computation of the emitted radiation.

In both cases, this calculation is directly based on Maxwell's equations, i.e. no approximations are made. Typically, the trajectories of the particles participating in the shower are approximated by line segments of sufficient granularity. The electric field $\boldsymbol{E}$ (or, alternatively, the vector potential $\boldsymbol{A})$ is known analytically for a particle travelling along a single such line segment, and so the total field distribution can be computed by superimposing all contributions.

This electric field configuration is then propagated through the environment in which the experiment is operating (which might be nontrivial, e.g. in the presence of varying atmospheric conditions or ice-air interfaces). Finally, the electric field at the location of the detector is passed through a simulation of the receiving antenna and the initial signal processing chain (such as linear filtering) to give access to the electric signal that forms the input for the actual reconstruction and subsequent data analysis.

In this contribution, we present an alternative approach for the computation of the signal, which, in some sense, proceeds in the inverse order. In our formalism, the detector is imagined to be the source of a particular electric field distribution that would ensue if a delta-like current was applied between the terminals of its antenna. This position- and time-dependent "weighting field" contains the full amount of information about the properties of the detector and its environment. From the statement of reciprocity, a central feature of classical electrodynamics, it follows that the signal produced in the detector by the shower can be computed very efficiently by convolving the weighting field with the trajectories of the participating particles. This procedure holds true in complete generality and is guaranteed to lead to consistent results for the induced signal, irrespective of the details of the input trajectories.

This correspondence is derived formally in Section 2 starting from Maxwell's equations. Section 3 then applies this formalism to the situation discussed above. More information about the method and other applications is available in Ref. [3].

\section{A general signal theorem for Maxwell's equation}

We begin by introducing a fundamental property of classical electromagnetism, the concept of reciprocity in Section 2.1. Section 2.2 then uses this result to derive a procedure for the computation of the induced detector signal.

\subsection{The concept of reciprocity}

We phrase our discussion in the context of a linear, anisotropic material distribution, which is general enough to encompass both the detector setup (antennas, cables, ... ) as well as its environ- 
a)

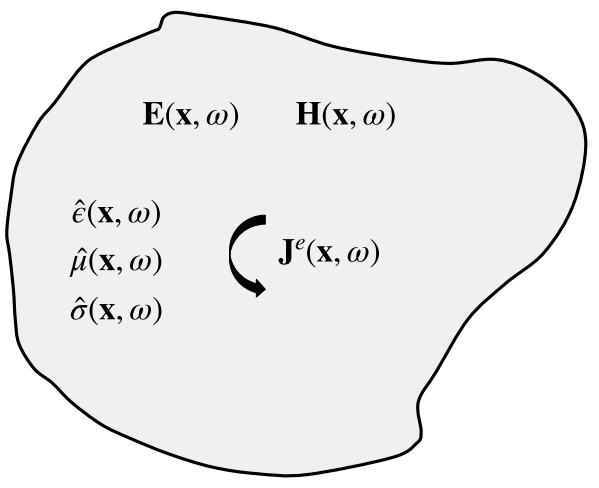

b)

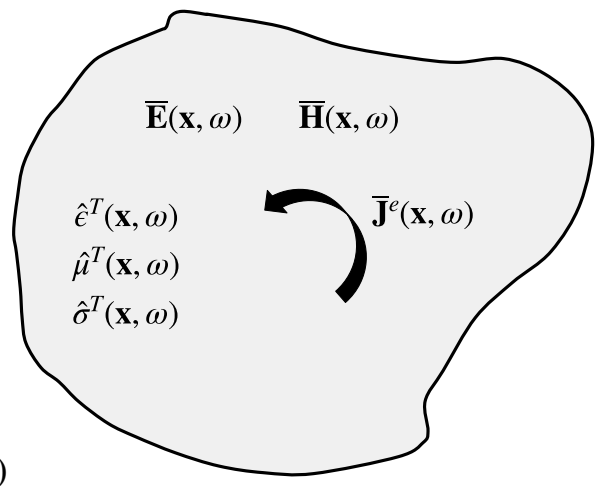

Figure 1: a) An external current $\boldsymbol{J}^{e}$ creates electric and magnetic fields $\boldsymbol{E}$ and $\boldsymbol{H}$ in a general linear material. b) A current $\overline{\boldsymbol{J}}^{e}$ creates fields $\overline{\boldsymbol{E}}$ and $\overline{\boldsymbol{H}}$ in a geometry with transposed material properties.

ment (atmosphere, ice, ... ). Such a material is characterised by a position- and frequency-dependent permittivity matrix $\hat{\boldsymbol{\varepsilon}}(\boldsymbol{x}, \omega)$, permeability matrix $\hat{\boldsymbol{\mu}}(\boldsymbol{x}, \omega)$ and conductivity matrix $\hat{\boldsymbol{\sigma}}(\boldsymbol{x}, \omega)$. These $3 \times 3$ matrices determine the constitutive equations of the material,

$$
D=\hat{\varepsilon} E, \quad B=\hat{\mu} H, \quad J=\hat{\sigma} E .
$$

As illustrated in Fig. 1a, an externally impressed current density $\boldsymbol{J}^{e}(\boldsymbol{x}, \omega)$ flowing through this material creates electric and magnetic fields $\boldsymbol{E}(\boldsymbol{x}, \omega)$ and $\boldsymbol{H}(\boldsymbol{x}, \omega)$. These field distributions are solutions of Maxwell's equations. In the Fourier domain, where $\frac{\partial}{\partial t} \equiv i \omega$, they read as follows,

$$
\begin{gathered}
\boldsymbol{\nabla} \cdot \hat{\boldsymbol{\varepsilon}} \boldsymbol{E}=\rho, \quad \boldsymbol{\nabla} \cdot \hat{\boldsymbol{\mu}} \boldsymbol{H}=0, \\
\boldsymbol{\nabla} \times \boldsymbol{E}=-i \omega \hat{\boldsymbol{\mu}} \boldsymbol{H}, \quad \boldsymbol{\nabla} \times \boldsymbol{H}=\boldsymbol{J}^{e}+\hat{\boldsymbol{\sigma}} \boldsymbol{E}+i \omega \hat{\boldsymbol{\varepsilon}} \boldsymbol{E} .
\end{gathered}
$$

We now consider the situation depicted in Fig. 1b, which is built around a different material distribution: it is obtained by transposing the original material properties, i.e. replacing $\hat{\varepsilon} \rightarrow \hat{\varepsilon}^{T}$, $\hat{\mu} \rightarrow \hat{\mu}^{T}$ and $\hat{\boldsymbol{\sigma}} \rightarrow \hat{\boldsymbol{\sigma}}^{T}$, but keeping their dependence on position and frequency unchanged. An external current density $\overline{\boldsymbol{J}}^{e}$ impressed on this material distribution leads to fields $\overline{\boldsymbol{E}}(\boldsymbol{x}, \omega)$ and $\overline{\boldsymbol{H}}(\boldsymbol{x}, \omega)$, which are again solutions of Maxwell's equations. In the remainder of this section, we formulate a precise relationship between these two situations, i.e. between $\boldsymbol{E}, \boldsymbol{H}, \boldsymbol{J}{ }^{e}$ on the one hand, and $\overline{\boldsymbol{E}}, \overline{\boldsymbol{H}}, \overline{\boldsymbol{J}}^{e}$ on the other hand.

To find such a connection, we make use of the product rule of calculus. Applied to two arbitrary vector fields $\boldsymbol{F}$ and $\boldsymbol{G}$, it states that

$$
\boldsymbol{\nabla} \cdot(\boldsymbol{F} \times \boldsymbol{G})=\boldsymbol{G} \cdot(\boldsymbol{\nabla} \times \boldsymbol{F})-\boldsymbol{F} \cdot(\boldsymbol{\nabla} \times \boldsymbol{G}) .
$$

This implies that

$$
\begin{aligned}
\boldsymbol{\nabla} \cdot(\boldsymbol{E} \times \overline{\boldsymbol{H}}) & =\overline{\boldsymbol{H}} \cdot(\boldsymbol{\nabla} \times \boldsymbol{E})-\boldsymbol{E} \cdot(\boldsymbol{\nabla} \times \overline{\boldsymbol{H}}) \\
& =-\boldsymbol{E} \cdot \overline{\boldsymbol{J}}^{e}-i \omega \overline{\boldsymbol{H}} \cdot \hat{\boldsymbol{\mu}} \boldsymbol{H}-\boldsymbol{E} \cdot\left(\hat{\boldsymbol{\sigma}}^{T}+i \omega \hat{\boldsymbol{\varepsilon}}^{T}\right) \overline{\boldsymbol{E}}
\end{aligned}
$$

and also

$$
\begin{aligned}
\boldsymbol{\nabla} \cdot(\overline{\boldsymbol{E}} \times \boldsymbol{H}) & =\boldsymbol{H} \cdot(\boldsymbol{\nabla} \times \overline{\boldsymbol{E}})-\overline{\boldsymbol{E}} \cdot(\boldsymbol{\nabla} \times \boldsymbol{H}) \\
& =-\overline{\boldsymbol{E}} \cdot \boldsymbol{J}^{e}-i \omega \boldsymbol{H} \cdot \hat{\boldsymbol{\mu}}^{T} \overline{\boldsymbol{H}}-\overline{\boldsymbol{E}} \cdot(\hat{\boldsymbol{\sigma}}+i \omega \hat{\boldsymbol{\varepsilon}}) \boldsymbol{E} .
\end{aligned}
$$


a)

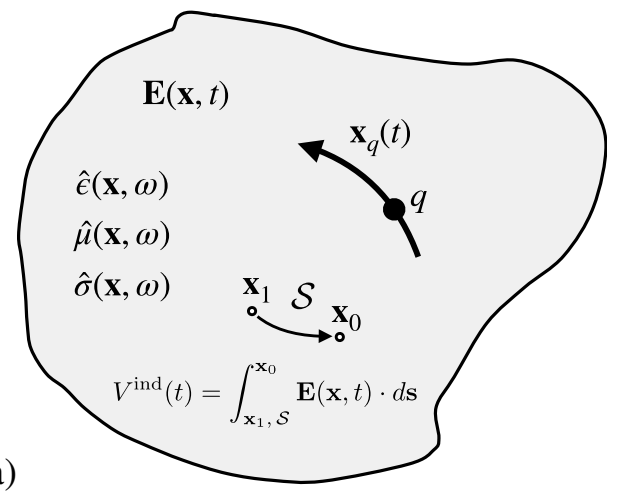

b)

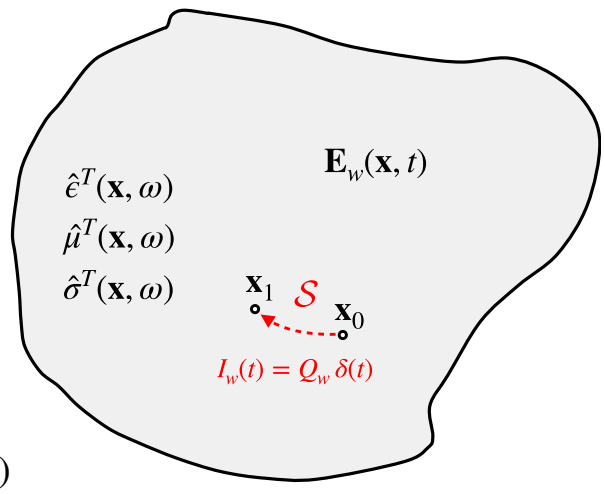

Figure 2: a) A moving point charge creates an electric field and therefore a voltage $V^{\text {ind }}$ between the points $\boldsymbol{x}_{0}$ and $\boldsymbol{x}_{1}$. b) A line current $I_{w}=Q_{w} \delta(t)$ generates the weighting field $\boldsymbol{E}_{w}(\boldsymbol{x}, t)$.

Subtracting these two expressions, we get

$$
\boldsymbol{\nabla} \cdot(\boldsymbol{E} \times \overline{\boldsymbol{H}}-\overline{\boldsymbol{E}} \times \boldsymbol{H})=\overline{\boldsymbol{E}} \cdot \boldsymbol{J}^{e}-\boldsymbol{E} \cdot \overline{\boldsymbol{J}}^{e} .
$$

Integrating this over an arbitrary volume $V$ and applying Gauss' theorem we have

$$
\int_{\partial V} d \boldsymbol{A} \cdot(\boldsymbol{E} \times \overline{\boldsymbol{H}}-\overline{\boldsymbol{E}} \times \boldsymbol{H})=\int_{V} d V\left(\overline{\boldsymbol{E}} \cdot \boldsymbol{J}^{e}-\boldsymbol{E} \cdot \overline{\boldsymbol{J}}^{e}\right)
$$

where $d \boldsymbol{A}$ is the surface element of the boundary of $V, A=\partial V$. We now take the limit where the boundary is pushed to infinity, i.e. the volume $V$ fills all of space. If the sources are compact, i.e. contained in a bounded spatial region, the surface term on the left-hand side evaluates to zero.

We thus find

$$
\int d V \overline{\boldsymbol{E}}(\boldsymbol{x}, \omega) \cdot \boldsymbol{J}^{e}(\boldsymbol{x}, \omega)=\int d V \boldsymbol{E}(\boldsymbol{x}, \omega) \cdot \overline{\boldsymbol{J}}^{e}(\boldsymbol{x}, \omega)
$$

which is known as the Lorentz reciprocity theorem [4]. This is the relation we were setting out to find: it will be central to the formulation of our signal theorem below.

\subsection{A general signal theorem}

We employ the Lorentz reciprocity relation to compute the signal produced in a general particle detector by a moving charge. As illustrated in Fig. 2a, we consider a point charge $q$ that moves along an arbitrary trajectory $\boldsymbol{x}_{q}(t)$. The current density created by this motion is given by

$$
\boldsymbol{J}^{e}(\boldsymbol{x}, t)=q \dot{\boldsymbol{x}}_{q}(t) \delta\left[\boldsymbol{x}-\boldsymbol{x}_{q}(t)\right] .
$$

We keep the material properties $\hat{\boldsymbol{\varepsilon}}, \hat{\boldsymbol{\mu}}$ and $\hat{\boldsymbol{\sigma}}$ completely general, as before. We arrange the detector such that it delivers its readout signal in the form of a voltage $V^{\text {ind }}(t)$ measured between the positions $\boldsymbol{x}_{1}$ and $\boldsymbol{x}_{0}$ along a particular path $\mathcal{S}$. We parametrise $\mathcal{S}$ as $\boldsymbol{x}_{s}(s)$ with $\boldsymbol{x}_{s}\left(s_{1}\right)=\boldsymbol{x}_{1}$ and $\boldsymbol{x}_{s}\left(s_{0}\right)=\mathbf{x}_{0}$. The signal in the frequency domain is then defined as

$$
V^{\mathrm{ind}}(\omega):=\int_{\boldsymbol{x}_{1}, \mathcal{S}}^{\boldsymbol{x}_{0}} d \boldsymbol{s} \cdot \boldsymbol{E}(\boldsymbol{x}, \omega)=\int_{s_{1}}^{s_{0}} d s \boldsymbol{E}\left(\boldsymbol{x}_{s}(s), \omega\right) \cdot \frac{d \boldsymbol{x}_{s}(s)}{d s} .
$$


Note that the specification of the path $\mathcal{S}$ is important: in the general case where $\boldsymbol{\nabla} \times \boldsymbol{E} \neq 0$, the signal $V^{\text {ind }}$ is not independent of the chosen path. In a traditional particle detector the point $\boldsymbol{x}_{1}$ typically represents the signal electrode and the point $\boldsymbol{x}_{0}$ corresponds to the ground reference.

We now consider a second scenario, shown in Fig. 2b. The point charge is removed and instead a line current $I_{w}(\omega)$ is set to flow from $\boldsymbol{x}_{0}$ to the detector terminal at $\boldsymbol{x}_{1}$ along the same path $\mathcal{S}$, but now embedded in the material distribution with transposed response matrices. The direction of the current is chosen for consistency with conventional definitions of the signal polarity. This current creates an electric field $\boldsymbol{E}_{w}$ (referred to as the "weighting field"), as determined by Maxwell's equations. Inserting the above expressions into the reciprocity relation in Eq. 1, we get

$$
V^{\text {ind }}(\omega)=-\frac{1}{I_{w}(\omega)} \int d V \boldsymbol{E}_{w}(\boldsymbol{x}, \omega) \cdot \boldsymbol{J}^{e}(\boldsymbol{x}, \omega)
$$

The current $I_{w}(\omega)$ is completely arbitrary. If we take it to be independent of the frequency $\omega$, it corresponds to a delta-like current $I_{w}(t)=Q_{w} \delta(t)$ in the time domain. We can then perform the inverse Fourier transform of Eq. 2 and find the induced signal in the time domain as

$$
V^{\text {ind }}(t)=-\frac{q}{Q_{w}} \int_{-\infty}^{\infty} d t^{\prime} \boldsymbol{E}_{w}\left(\boldsymbol{x}_{q}\left(t^{\prime}\right), t-t^{\prime}\right) \cdot \dot{\boldsymbol{x}}_{q}\left(t^{\prime}\right)
$$

This formula is the core of the following procedure for the computation of the signal. The induced voltage $V^{\text {ind }}(t)$ measured along a path $\mathcal{S}$ from $\boldsymbol{x}_{1}$ to $\boldsymbol{x}_{0}$ that is induced by the movement of a point charge q along a trajectory $\boldsymbol{x}_{q}(t)$ through a material with parameters $\hat{\boldsymbol{\varepsilon}}(\boldsymbol{x}), \hat{\boldsymbol{\mu}}(\boldsymbol{x}), \hat{\boldsymbol{\sigma}}(\boldsymbol{x})$ can be calculated in the following way. The charge $q$ is removed and a delta current pulse $Q_{w} \delta(t)$ is placed along $\mathcal{S}$ flowing from $\boldsymbol{x}_{0}$ to $\boldsymbol{x}_{1}$ through a material with parameters $\hat{\boldsymbol{\varepsilon}}(\boldsymbol{x})^{T}, \hat{\boldsymbol{\mu}}(\boldsymbol{x})^{T}, \hat{\boldsymbol{\sigma}}(\boldsymbol{x})^{T}$. The response to this current is the electric weighting field $\boldsymbol{E}_{w}(\boldsymbol{x}, t)$. The detected voltage signal can be calculated by convolving the weighting field with the velocity of the particle according to Eq. 3 .

The above signal theorem is completely general, and only requires that the detector be made from linear materials. Many practically relevant situations are indeed already described well by symmetric material parameters. Nonlinear materials and materials exposed to fixed background fields can be treated with the same formalism in excellent approximation, as explained in Ref. [3].

The weighting field $\boldsymbol{E}_{w}(\boldsymbol{x}, t)$ makes no reference to the trajectory of the moving particle, but is a sole property of the detector and its environment. It can be computed once and for all for a given geometry, which is even possible analytically in simple cases. For realistic scenarios, standard numerical solvers can be used instead, and the weighting field distribution $\boldsymbol{E}_{w}(\boldsymbol{x}, t)$ saved to disk. To obtain the induced signal, it is then sufficient to perform a simple (numerical) convolution with the particle trajectory.

\subsection{Signal processing}

The raw detector signal $V^{\text {ind }}(t)$ is usually fed into an amplifier or a general linear signal processing chain with transfer function $F(\omega)$, which delivers the (amplified and filtered) signal $V^{\text {out }}(t)$ at its output.

Eqs. 2 and 3 imply that the filtered signal can be computed directly, without first having to compute the raw signal, or indeed the electric field at the position of the detector. Provided that the weighting field $\boldsymbol{K}_{w}(\boldsymbol{x}, \omega)$ is now suitably constructed as the result of a current $I_{w}(\omega)=Q_{w} F(\omega)$ 


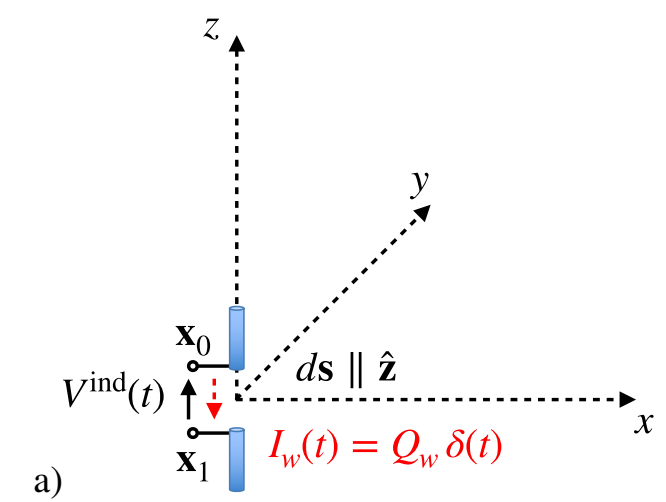

b)

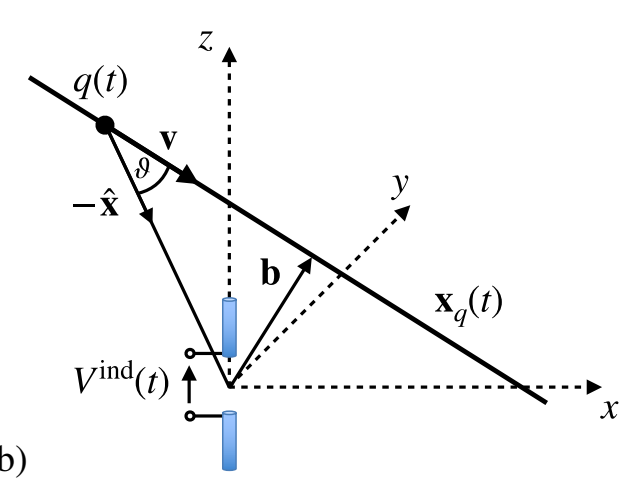

Figure 3: a) An infinitesimal electric dipole as particle detector. b) A simple analytic model of an extended air shower whose radio emission is measured by the dipole.

applied to the readout electrodes in the way shown above, the filtered signal is given by the convolution

$$
V^{\mathrm{out}}(t)=-\frac{q}{Q_{w}} \int_{-\infty}^{\infty} d t^{\prime} \boldsymbol{K}_{w}\left(\boldsymbol{x}_{q}\left(t^{\prime}\right), t-t^{\prime}\right) \cdot \dot{\boldsymbol{x}}_{q}(t)
$$

In practice, the bandwidth of the weighting field $\boldsymbol{K}_{w}$ is limited by $F(\omega)$. It is thus much easier to obtain numerically than the unfiltered weighting field $\boldsymbol{E}_{w}$.

\section{Applications and use cases}

In general, the characteristics of the raw detector signal $V^{\text {ind }}(t)$ depend in a complicated way on the geometry of the antenna configuration and the electric field in the vicinity of the detector. Below, we consider a simple application to illustrate our approach and to show its equivalence to established methods.

\subsection{Infinitesimal electric dipole: a simple detector}

We can form an infinitesimal electric dipole-indeed one of the simplest conceivable radio detectors - by separating the points $\boldsymbol{x}_{0}$ and $\boldsymbol{x}_{1}$ by an infinitesimal distance $d s$ along the $z$-axis, depicted in Fig. 3a. As shown in Ref. [3], the induced signal is directly proportional to the electric field at the position of the antenna, i.e. $V^{\text {ind }} \propto d \mathbf{s} \cdot \boldsymbol{E}$. The infinitesimal electric dipole thus provides a useful calculational vehicle to find the electric field at the location of a (more complicated) detector.

The weighting field $\boldsymbol{E}_{w}$ is the result of a line current applied between the terminals of the antenna, as indicated by the red arrow in Fig. 3a. For this simple case, it can be computed analytically. In polar coordinates, it reads

$$
\begin{aligned}
& E_{w}^{\theta}(r, \theta)=-\frac{Q_{w} d s}{4 \pi \varepsilon} \frac{\sin \theta}{r^{3}}\left[\Theta\left(t-\frac{r n}{c}\right)+\frac{r n}{c} \delta\left(t-\frac{r n}{c}\right)+\left(\frac{r n}{c}\right)^{2} \delta^{\prime}\left(t-\frac{r n}{c}\right)\right], \\
& E_{w}^{r}(r, \theta)=-2 \frac{Q_{w} d s}{4 \pi \varepsilon_{0}} \frac{\cos \theta}{r^{3}}\left[\Theta\left(t-\frac{r n}{c}\right)+\frac{r n}{c} \delta\left(t-\frac{r n}{c}\right)\right], \\
& E_{w}^{\phi}(r, \theta)=0,
\end{aligned}
$$


where $\Theta(x)$ is the Heaviside step function and $\delta^{\prime}(x)$ is the distributional derivative of the Dirac delta distribution. The quantity $r$ labels the radial distance from the antenna, $\theta$ is the polar angle measured from the $z$-axis, and $\phi$ is the azimuthal angle measured from the $x$-axis. The propagation speed of the shock front is given by $v=c / n$ where $n=(c \sqrt{\mu \varepsilon})^{-1}$ is the refractive index of the material and $c$ is the speed of light in vacuum.

\subsection{Signal from a simple one-dimensional shower}

To put this result to use, we consider a simple one-dimensional air shower in the situation depicted in Figure $3 b$. We take a point charge to move along a linear trajectory $\boldsymbol{x}_{q}(t)=\boldsymbol{b}+\boldsymbol{v} \cdot \boldsymbol{t}$ with $\boldsymbol{v}=\boldsymbol{\beta}$ c. To model the increasing negative charge excess in the shower front (electrons from the surrounding air being scattered into the shower, and positrons annihilating), we take the point charge $q(t)$ to depend explicitly on time.

Performing the convolution of the weighting field in Eq. 4 with the shower trajectory allows the electric field at the position of the dipole antenna to be determined. We find

$$
\begin{aligned}
\boldsymbol{E}(t)= & -\frac{d s}{4 \pi \varepsilon}\left[\frac{q(t)}{|1-n \beta \cos \vartheta|^{3}} \frac{1}{r^{2}}\left(1-n^{2} \beta^{2}\right)(\hat{\boldsymbol{x}}+n \beta)\right]_{t_{\mathrm{ret}}} \\
& -\frac{d s}{4 \pi \varepsilon}\left[\frac{\dot{q}(t)}{|1-n \beta \cos \vartheta|(1-n \beta \cos \vartheta)} \frac{n}{r c}(n \beta-(n \beta \cdot \hat{\boldsymbol{x}}) \hat{\boldsymbol{x}})\right]_{t_{\mathrm{ret}}} \\
& +\frac{d s}{4 \pi \varepsilon} \int_{-\infty}^{t_{\mathrm{ret}}} d t^{\prime} \dot{q}\left(t^{\prime}\right) \frac{\boldsymbol{x}_{q}\left(t^{\prime}\right)}{r^{3}}
\end{aligned}
$$

where $\vartheta$ is the angle between $\boldsymbol{v}$ and $-\hat{\boldsymbol{x}}$ and the retarded time $t_{\text {ret }}$ is defined by $t_{\text {ret }}=t-\frac{\left\|\boldsymbol{x}_{q}\left(t_{\text {ret }}\right)\right\|}{c / n}$. The first line in Eq. 5 is recognised as the Lorentz-boosted Coulomb field of the moving charge $q(t)$. The second line is proportional to $\dot{q}(t)$ and corresponds to the Askaryan radiation. The obtained expression is identical to the one computed in Ref. [5] with other methods. Finally, the third line implements the Coulomb field of the positive ions that are left behind by the shower. The presence of this contribution demonstrates that charge conservation is automatically imposed, and the electric field computed with our method is manifestly consistent.

\section{Conclusions}

The method of weighting fields provides an appealing procedure for the calculation of electric signals (or electric field) that are induced in a detector by a cosmic ray-induced shower. The weighting field is the electric field distribution that is produced when the detector is (imagined to be) operated as a transmission device. Convolving this field distribution with the trajectories of the moving charges in the shower then produces a consistent solution of Maxwell's equations.

For simple geometries, the weighting field can be computed analytically, which shows that our method is mathematically equivalent to the algorithms employed in modern Monte Carlo simulation tools. For realistic scenarios, the weighting field can be obtained through numerical solvers once and for all, and then used for the computation of the signal from arbitrary showers. It encodes the properties of the detector, its signal processing chain, as well as the environment in which it operates in a well-defined way. This ensures a transparent design of the simulation chain, and 
allows to implement refinements in the model simply by updating the weighting field and without modifications to the simulator code as such.

\section{References}

[1] M. Ludwig, T. Huege, REAS3: Monte Carlo simulations of radio emission from cosmic ray air showers using an "end-point" formalism, Astropart. Phys., 34 438-446 (2011)

[2] J. Alvarez-Muniz, W. R. Carvalho Jr., E. Zas, Monte Carlo simulations of radio pulses in atmospheric showers using ZHAireS, Astropart. Phys. 35, 325-341 (2012)

[3] W. Riegler, P. Windischhofer, Signals induced on electrodes by moving charges, a general theorem for Maxwell's equations based on Lorentz-reciprocity, Nucl. Instrum. Meth. A 980, $164471(2020)$

[4] H. A. Lorentz, Het theorema van Poynting over energie in het electromagnetisch veld en een paar algemeene stellingen over de voorplanting van het licht, Vers. Konig. Akad. Wetensch. 4, $176(1896)$

[5] J. Alvarez-Muniz, A. Romero-Wolf, E. Zas, Cerenkov radio pulses from electromagnetic showers in the time domain, Phys. Rev. D 81, 123009 (2010) 